\title{
Comparing Chinese and American oncology practice patterns using precision therapy for non-small cell lung
}

\section{cancer}

\author{
Zhang Ming ${ }^{1}$, Hao Xinbao ${ }^{2}$, Han Liang Fu ${ }^{3}$, Hu Honglin ${ }^{1}$, Ao Rui ${ }^{1}$, Zhu Xueqiang ${ }^{1}$, He Yangke ${ }^{1}$, Chen Lin ${ }^{1}$ and Ming Zeng',4* \\ ${ }^{1}$ Cancer Center, Sichuan Provincial Hospital Cancer Center, The Affiliated Hospital of the University of Electronic Science and Technology of China, China \\ ${ }^{2}$ China-America Cancer Center, Hainan Medial University, China \\ ${ }^{3}$ Department of Oncology, ChangAn Hospital, China \\ ${ }^{4}$ Department of Hematology and Oncology, Zangmeister Cancer Center, Mount Carmel Health System, USA
}

\begin{abstract}
Background: Little research has compared lung cancer-related clinical practice patterns at different geographic locations. This study was to explore the understandings and progress of precision therapy application in non-small cell lung cancer (NSCL) among oncologists at different geographic locations in China and the United States.

Methods: A survey of oncologists at four different locations, three in China and one in the U.S to explore their understandings of precision oncology in NSCL and their practice patterns.

Results: 77 (82\%) valid responds were received, including 53 from China and 24 from the United States. There was no significant difference among Chinese oncologists at the three different Chinese facilities in their understandings of the molecular mechanism, needs for companion diagnosis in initiate treatment, stage requirement for the treatment, the cost of precision components, treatment strategy, molecular prevention concept and side effects from precision oncology. But Chinese oncologists in tertiary facilities recognized more problems of drug resistance compared with those in community facilities ( $\mathrm{p}=0.02$ ). Between oncologists in the two countries, there were significant differences in recognizing the side effects $(\mathrm{p}=0.03$ ), improvement in only DFS not OS ( $\mathrm{p}=0.002$ ), and drug resistance $(\mathrm{p}=0.01)$. Chinese oncologists expected more enrollments in clinical studies, while American oncologists wanted more high quality practice change studies.
\end{abstract}

Conclusions: All respondents were good at complying with available precision oncology guideline. Oncologists from China and the United States had different focuses in clinical studies that reflected differences in oncological infrastructures between these two countries.

\section{Introduction}

Lung cancer is the most deadly cancer in either the US or China. How to improve treatment outcomes is a major concern for oncologists in their daily practice. Oncologists are generally optimistic about the applications of new technology in their practice, with a hope of improving the outcomes in their practice. One key issue is how quickly new research findings, e.g., the application of small molecular target agent or immunotherapy in lung precision oncology, can be translated from bench to bedside. Personalized medicine in oncology based upon individual tumor molecular specificity has been successful implemented in precision oncology [1]. Clinical compliance to the fast changing precision oncology presents a challenge all oncologists. The compliance of updated precision oncology depends on the readiness of health care practitioners to adopt the technology, along with the availability of the needed infrastructures.

There has been a tremendous paradigm shift in the evaluation and treatment of lung cancer over the last decade. Discovery of an exquisitely sensitive treatment to those oncogenic drivers such as agents mutated EGFR gene or gene rearrangements in ALK in NSCL expanded the frontiers of precision oncology. These genomic subsets have a distinct clinical pathologic presentation, natural history, and disease course, and they under certain special conditions are associated with double lung cancer patients' survival [2]. However, phase 3 studies have not repeated the survival benefits. Moreover, a recent report was skeptical about the over-optimism about the bench-to-bed translation process. Unlike other technology, one report suggested that the reality of precision oncology was a fast translating in mind for practitioners rather than quick bringing in outcomes for lung cancer patients [3].

To evaluate oncologists' basic conceptual understandings of the target therapy and its clinical applications, we conducted a survey among different groups of physicians in two countries, China and the United States from April $10^{\text {th }}$ to May $20^{\text {th }}, 2015$. The participants were asked to answer the survey questions to their best knowledge. We assumed that there could be differences among facilities or locations

Correspondence to: M Zeng, Sichuan Provincial Hospital Cancer Center, the Affiliated Hospital of the University of Electronic Science and Technology of China, Chengdu, Sichuan 610072, China; E-mail: miller2002@yahoo.com

Key words: precision medicine, lung cancer, target therapy, clinical practice pattern

Received: October 11, 2015; Accepted: October 28, 2015; Published: October 31, 2015 
or countries in term of the oncologists' readiness in implementing precision oncology.

\section{Materials and methods}

In April 2015, we launched the survey among three different hospitals in China and one cancer center in the US. Among the three Chinese locations, two are tertiary hospitals with strong academic activity, and one of these two hospitals is the national center that conducts the from-bench-to-bed translational study. The third Chinese hospital is a community hospital. A total of 94 requests were sent out to the Chinese and American sites, and a total of 77 valid responses were received, resulting in an $82 \%$ response rate. Participants completed the survey either via electronically or on paper copies. The data were entered into a spreadsheet table for analysis. Data collection lasted for one month.

We had items to collect information about the participants' lung cancer patient management background and related information as characteristic variables. To quantify the prevalence in these oncologists' understanding of the mechanism of precision oncology, as opposed to more deliberate decision-making, we asked the participants to answer more clinically relevant questions. We also asked questions about common phenomena in their practice such as drug resistance and its mechanism, and related resolution. In treatment indication, we asked questions about cancer stage and its application indications, with open-end questions for those who would like to answer more specific histology subtypes, or clinical situation related treatment indications, expectation from treatments.

To investigate current clinical study platform, we asked the participants questions about their satisfaction towards the clinical study infrastructure, such as protocol access, trial enrollment and data publication.

We are more inclusive of all those readable data, no biased selection. We calculated descriptive statistics, and used Chi-Square tests to compare the results among sites.

\section{Results}

A total of 77 participants answered all questions in the questionnaire; those who did not were excluded from further analysis. Among the 77 completed questionnaires, 53 were from China and 24 were from the US. Of the 53 responses from China, 27 were from tertiary hospitals and 26 were from a community hospital. Among the 77 participants who completed the questionnaire, $89 \%$ of the participants from China were medical oncologists, while $77 \%$ of the participants from the US were medical oncologists. The majority of these medical oncologists were managing lung cancer patients (Table 1).

\section{Companion pathological diagnostics}

Oncologists from both countries and all locations were aware of the necessity of companion pathological test for precision oncology. However, there were differences in their practice patterns. In China, there was less restriction for molecular pathology test and offering off protocol target agents treatment upfront. In comparison, the US oncologists would have to wait until third party approval before staring the test and treatment. The timing for starting the treatment was critical in NSCLC, where up to one-third of patients may have upfront mutations. If an oncologist couldn't use precision agents in first-line therapy, then s/he often would start with the next line therapy that could result in the heterogeneity changes of the cancer tissue, making the initial pathological diagnosis less reliable. The timing to return for report also is a concern in the US. Some tests take time-up to several weeks-but many cancer patients can't wait that long for a new therapy. The timing for return report is less of a concern in China, in comparison. A common concern in both countries was acquiring enough tissue for appropriate testing. Technology was better, but we still needed tumor tissue for testing. In some situations, more tissue was not available, and an additional biopsy might be required.

All participants expressed concerns about the less well defined test market where some vendors have more updated sequencing experience than others. Participants also needed to coordinate with the laboratory and pathologists.

Then there is the matter of interpretation: "What do the tests mean??" Labs were getting better at providing annotated reports to help oncologists interpret data. The results were still complex and many clinicians do not understand the significance. Using the information to treatment decisions can be difficult for a busy practitioner who may not have the time or resources to fully interpret the reports, adding that many laboratories now asked for more molecular geneticists.

All respondents complied with the updated ASCO guidelines, which recommend adenocarcinoma or mixed with adenocarcinoma component to be tested for EGFR and ALK mutation at the time of diagnosis or recurrence. The guidelines did not recommend routing test for KRAS mutation, stating that "that testing for KRAS mutations may be performed initially to eliminate the need to probe for $E G F R$ and $A L K$ alterations, which are mutually exclusive with KRAS".

\section{Side effects}

Among the Chinese sites, the concern for precision medicine toxicity was low. There were more concerns for the toxicity from target therapy or immunotherapy among American oncologists compared with those in China $(\mathrm{p}=0.03)$.

Table 1. Characteristics of survey participants.

\begin{tabular}{|l|c|c|c|}
\hline \multirow{2}{*}{} & \multicolumn{3}{|c|}{ Analytic sample characteristics, number } \\
\cline { 2 - 4 } & \multicolumn{2}{|c|}{ China } \\
\cline { 2 - 4 } & Facility One, Tertiary & Facility Two, Tertiary & Facility Three, Community \\
\hline Oncologist & 15 & 12 & 26 \\
\hline Medical Oncologist & 15 & 12 & 20 \\
\hline Non-Medical Oncologist & 0 & 0 & 6 \\
\hline Managed Lung Cancer & 15 & 12 & 24 \\
\hline Do not Manage Lung Cancer & 0 & 0 & 12 \\
\hline Total & 15 & 12 & 24 \\
\hline
\end{tabular}


US oncologists were less optimistic about the promise of precision oncology. They were concerns about having only few successful targets agent being able to achieve expected positive results, the cost of the agents, and unexpected side effects all being well balanced and recognized. In short, the US oncologists seemed more down to the reality, rather than being over-optimistic about precision medicine.

\section{Reimbursement}

Reimbursement policies are different in China and the United States. More people in China are willing to pay cash if a third party denied payment for molecular diagnosis and target agents of precision medicine. US oncologists cited education as a necessity to achieve full implementation of personalized medicine. However, some of them also saw it as an impediment to expanded use. Both reimbursement and funding are necessary for broader use of precision medicine.

In the US, the value assessment essential for reimbursement is driven by value. With more clinical trial data, insurance companies have been receptive to molecular diagnosis, which is one of the high cost burdens for both patients and insurance companies $[4,5]$. The regulation for precision medicine was getting more restricted with large usage and less than expected results. The bottom line was that oncologists from both countries and different locations within China had a consensus for the high cost for precision oncology (Table 2b).

\section{DFS not OS}

The biggest flaw in precision medicine is that the short lasting results, that only presented as DFS or PFS but not overall survival. The benefits thus far tend to be very short-lived before other mutations emerge and disease control is lost. Cure was virtually not reported, but before the resistance is overcome, the best results would be improvement of DFS. Median disease control is usually measured in months, and rarely approached anything close to a year. As such, patients were too frequently experiencing an 'is-that-all-there-is' phenomenon. When they get precision medicine, all too soon they are looking into the abyss again. There were significant differences in recognizing the ultimate results from precision oncology treatment; American Oncologists had more knowledge about the goal of the treatment than their counterparts in China, where oncologists within various locations and service levels in China were more optimistic about the target agents (Table 2b).

\section{Drug resistance}

All oncologists were aware of drug resistance (Table 2a). Unless another success story like imatinib in CML merged, the challenges were to target tumor resistance. The reality in lung cancer is that cancer can develop through multiple abnormal pathways and at multiple points within a single pathway. Some "smart cancers" can mutate and create "escape routes" through new pathways, which can lead to drug resistance. A strategy of combination targeted therapies may block multiple targets, stopping escape routes, preventing resistance, and improving outcomes.

\section{Trial vs. standard care}

The indications for precision medicine required more data from molecular pathology. A basic question for oncologists to think about was how to use the target agents in their daily practice. There was no significant difference among different locations within China, or between the two countries in general, where the practice was based on available clinical data. However, there is difference among the

Table 2a. The survey report from the facilities within China.

\begin{tabular}{|c|c|c|c|c|c|c|c|}
\hline \multicolumn{2}{|l|}{ Responses to the questions } & \multicolumn{4}{|c|}{ China } & \multicolumn{2}{|c|}{ Chi-Square Tests } \\
\hline & & \multirow{2}{*}{$\begin{array}{c}\begin{array}{c}\text { Facility One \& } \\
\text { Two, Tertiary }\end{array} \\
27\end{array}$} & \multirow{2}{*}{$\begin{array}{c}\% \\
100.00 \%\end{array}$} & & & & \\
\hline Precision Oncologist Knowledge & & & & $\begin{array}{c}\text { Facility Three, } \\
\text { Community }\end{array}$ & $\begin{array}{c}\% \\
100.00 \%\end{array}$ & $\begin{array}{c}\text { Value } \\
\mathrm{n} / \mathrm{a}^{*}\end{array}$ & $\begin{array}{c}\mathbf{P} \\
\mathrm{n} / \mathrm{a}\end{array}$ \\
\hline \multicolumn{8}{|l|}{ Clinic Indications } \\
\hline & stage indication & 25 & $92.59 \%$ & 20 & $76.92 \%$ & 1.462 & 0.227 \\
\hline Companion Pathology & & 24 & $88.89 \%$ & 23 & $88.46 \%$ & 0.000 & 1.000 \\
\hline \multicolumn{8}{|l|}{ side effects } \\
\hline & significant & 20 & $74.07 \%$ & 14 & $53.85 \%$ & 2.357 & 0.125 \\
\hline & not a concern & 0 & $0.00 \%$ & 3 & $11.54 \%$ & 1.495 & 0.221 \\
\hline Financial Issue & & 27 & $100.00 \%$ & 21 & $80.77 \%$ & 3.703 & 0.054 \\
\hline cost & concern & 21 & $77.78 \%$ & 20 & $76.92 \%$ & 0.006 & 0.941 \\
\hline \multicolumn{8}{|l|}{ Outcome } \\
\hline \multirow[t]{3}{*}{ Curable Intent } & yes & 8 & $29.63 \%$ & 4 & $15.38 \%$ & 1.535 & 0.215 \\
\hline & improve DFS & 6 & $22.22 \%$ & 6 & $23.08 \%$ & 0.006 & 0.941 \\
\hline & improve OAS & 1 & $3.70 \%$ & 1 & $3.85 \%$ & 0.000 & 1.000 \\
\hline Access to The Clinical Trial & & $\mathrm{N} / \mathrm{A}^{* *}$ & $\mathrm{~N} / \mathrm{A}$ & 5 & $19.23 \%$ & N/A & N/A \\
\hline Off Protocol Treatment & & N/A & N/A & 7 & $26.92 \%$ & N/A & N/A \\
\hline Protocol Availability & & & N/A & 9 & $34.62 \%$ & N/A & N/A \\
\hline Knowledge of Drug Resistance & & 26 & $96.30 \%$ & 18 & $69.23 \%$ & 5.097 & 0.024 \\
\hline Molecular Prevention Concept & & 1 & $3.70 \%$ & 1 & $3.85 \%$ & 0.000 & 1.000 \\
\hline Total & & 27 & $100.00 \%$ & 26 & $100.00 \%$ & & \\
\hline
\end{tabular}


Table 2b. The survey report from community hospital comparison between two counties.

\begin{tabular}{|c|c|c|c|c|c|c|c|}
\hline \multicolumn{2}{|c|}{ Responses to the questions } & \multicolumn{4}{|c|}{ Community Hospital } & \multicolumn{2}{|c|}{ Chi-Square Tests } \\
\hline & & \multirow{2}{*}{$\begin{array}{c}\text { China } \\
26\end{array}$} & \multirow{2}{*}{$\begin{array}{c}\mathbf{\%} \\
100.00 \%\end{array}$} & \multirow{2}{*}{$\begin{array}{l}\text { US } \\
24\end{array}$} & \multirow{2}{*}{$\begin{array}{c}\% \\
100.00 \% \\
\end{array}$} & \multirow{2}{*}{$\begin{array}{c}\text { Value } \\
\text { n/a }\end{array}$} & \multirow{2}{*}{$\begin{array}{c}\mathbf{P} \\
\mathrm{n} / \mathrm{a}^{*}\end{array}$} \\
\hline Precision Oncologist Knowledge & & & & & & & \\
\hline \multicolumn{8}{|l|}{ Clinic Indications } \\
\hline & stage indication & 20 & $76.92 \%$ & 22 & $91.67 \%$ & 1.071 & 0.301 \\
\hline Companion Pathology & & 23 & $88.46 \%$ & 22 & $91.67 \%$ & 0.000 & 1.000 \\
\hline \multicolumn{8}{|l|}{ side effects } \\
\hline & significant & 14 & $53.85 \%$ & 20 & $83.33 \%$ & 4.987 & 0.026 \\
\hline & not a concern & 3 & $11.54 \%$ & 0 & $0.00 \%$ & 1.255 & 0.263 \\
\hline Financial Issue & & 21 & $80.77 \%$ & 24 & $100.00 \%$ & 3.214 & 0.073 \\
\hline cost & concern & 20 & $76.92 \%$ & 17 & $70.83 \%$ & 0.241 & 0.624 \\
\hline \multicolumn{8}{|l|}{ Outcome } \\
\hline \multirow[t]{3}{*}{ Curable Intent } & yes & 4 & $15.38 \%$ & 0 & $0.00 \%$ & 2.195 & 0.138 \\
\hline & improve DFS & 6 & $23.08 \%$ & 16 & $66.67 \%$ & 9.624 & 0.002 \\
\hline & improve OAS & 1 & $3.85 \%$ & 0 & $0.00 \%$ & 0.000 & 1.000 \\
\hline Access to The Clinical Trial & & 5 & $19.23 \%$ & 24 & $100.00 \%$ & 33.422 & 0.000 \\
\hline Off Protocol Treatment & & 7 & $26.92 \%$ & 2 & $8.33 \%$ & 1.798 & 0.180 \\
\hline Protocol Availability & & 9 & $34.62 \%$ & 24 & $100.00 \%$ & 23.776 & 0.000 \\
\hline Knowledge of Drug Resistance & & 18 & $69.23 \%$ & 24 & $100.00 \%$ & 6.651 & 0.010 \\
\hline Molecular Prevention Concept & & 1 & $3.85 \%$ & 1 & $4.17 \%$ & 0.000 & 1.000 \\
\hline Total & & 26 & $100.00 \%$ & 24 & $100.00 \%$ & & \\
\hline
\end{tabular}

availabilityof the clinical trials between the countries; there is missed data for two locations within China regarding the availability for clinical trials due to missing data. It seemed that there were more clinical trials in precision medicine in community in the US comparing with its counterparts in China (Table 2b). Both TAPUR and NCI March trials were good examples. There was no large scare, multicenter trials in China for NSCL to author's knowledge.

\section{Current system effectiveness}

The oncologists from both countries felt that there was more work to do regarding the infrastructure. Both sides were skeptical of the current system, demanding more effective health care systems $(70 \%$ require). Both sides expected that personalized medicine would have a positive impact on lung cancer care.

\section{Molecular prevention}

Widely lacking basic understanding of molecular prevention existed in both countries. It seemed to be the last thing oncologists were aware of (Table 2a). The importance of further understanding the molecular onco-genesis could not be emphasized more. The onco-genesis would make a great success once the cause of molecular alteration and its impact on invasive and metastatic disease were well defined. The intervention before disease development is the best strategy from precision oncology.

\section{Discussion}

We conducted a survey to reveal oncological practice patterns in different locations in China and one location in the US. Our findings suggest that there were similar and different practice patterns in lung cancer precision management. The consensus appeared in indications, pathological requirement, the cost, and resistance of usage of special target agents in adenocarcinoma. Oncologists from both countries recognized the necessity of pathological requirement for special target agents' treatment. Our study provided evidence that the majority of oncologists closely followed up with various guidelines such as the NCCN guidelines to practice according established evidences. Some questions were raised for the companion pathology requirement, such as the sample volume, the biopsy timing, controversial for re-biopsy during treatment.

Regarding the outcome of precision oncology, most studies found any improvements were at the level of DFS. There are several potential reasons to explain the disassociation between improvements of DFS but not OS in precision oncology treatment for NSCL cancer. First, the therapeutic ratio was low, the benefits from precision medicine were counteracted by its side effects, even the target agents were clinical well tolerated, but asymptomatic toxicity could exist. The positive tumor control and its potential lifesaving capacity were balanced out by the negative impacts on patients overall. Second, due to quick establishment of target agents' resistance from the recolonization of the tumor, the positive lifesaving impact did not independently function before its efficacy downturned by the resistance. Finally, there might be systematic errors in our analysis. New analytical approach was proposed by others to sample less for individual's outcomes in precision medicine. An ' $n$ of 1' data or registry approach has been proposed [6]. The current clinical trial system doesn't allow drugs to be tested precisely; the OS benefit happened to individual was pooled and diluted in large scar trials. Before precision oncology, lung cancer was the number one cause of cancer death in both China and the United States [7] (Force). We should not lose sight of the fact that it still is. 
There is still a long way ahead of us to bring the mortality rate down through target agents for lung cancer.

Besides the similar practice patterns, we noticed the differences on the access the clinical study or enrollment among oncologists between the two countries. We provided evidence that absolute needs for large clinical trials infrastructure investment in China, the investments could come from private investigators such pharmaceutic companies (NCIMatch and TAPUR trials are sponsored by drug companies and patient receive drugs or some molecular tests without charge) or government fund, to established organization at various level institutes.

Public awareness and easy access to clinical trials is one of the differences between the two countries. Among the 3-level medical centers in China, the common answer for target agents treatment was either offering off protocol treatment or no treatment. Accessible clinical studies could be something that Chinese oncologists would love to consider. The importance of clinical trials was not only for the patient to access new, potential drugs without cost, but also to generate new data for future patient benefits. The infrastructure update in clinical research was a key to ensure that patients have access innovations in China.

Prospectively, the needs for more collaboration among different oncological fields never were so important, not only between pathologist and medical oncologist, but also all other oncological specialties. The systematic infrastructure to establish platforms such as multidisciplinary tumor board for discuss cases and clinical studies is crucial for this kind of collaborations.

\section{Conclusion}

This was a small sample study, not representative of broad practice patterns. All oncologists from various locations within China or between the countries were good at complying guidelines in precision oncology in their daily practice for NSCL patients. The high cost and limited efficacy for precision oncology were widely recognized. Oncologists from China expected more access to clinical trials instead of offering target components off protocols, and oncologists from the US expected more practice pattern changed high quality clinical trials data.

\section{References}

1. Garber K (2015) Oncologists push beyond new lung cancer genomic testing guidelines. $J$ Natl Cancer Inst 107. [Crossref]

2. Sellmann L, Fenchel K, Dempke WC (2015) Improved overall survival following tyrosine kinase inhibitor treatment in advanced or metastatic non-small-cell lung cancer-the Holy Grail in cancer treatment? Transl Lung Cancer Res 4: 223-227. [Crossref]

3. Jameson JL, Longo DL (2015) Precision medicine--personalized, problematic, and promising. N Engl J Med 372: 2229-2234. [Crossref]

4. Himmelstein DU, Thorne D, Warren E, Woolhandler S (2009) Medical bankruptcy in the United States, 2007: results of a national study. Am J Med 122: 741-746. [Crossref]

5. Neubauer MA, Hoverman JR, Kolodziej M, Reisman L, Gruschkus SK, et al. (2010) Cost effectiveness of evidence-based treatment guidelines for the treatment of nonsmall-cell lung cancer in the community setting. J Oncol Pract 6: 12-18. [Crossref]

6. Schork NJ (2015) Personalized medicine: Time for one-person trials. Nature 520: 609611. [Crossref]

7. Force, U.S. Preventative Services Task. "Lung Cancer: Screening. U.S. Preventative Services Task. Force website. [uspreventiveservicestaskforce.org/Page/Topic/ recommendation-summary/lung-cancer-screening."]. Accessedon March 21, 2015.

Copyright: (C) 2015 Ming Z. This is an open-access article distributed under the terms of the Creative Commons Attribution License, which permits unrestricted use, distribution, and reproduction in any medium, provided the original author and source are credited. 